\title{
Improved DV-HOP localization algorithm in the park location Guoqing $\mathrm{Qiu}^{\mathrm{a}}$, Jian $\mathrm{Wu}^{\mathrm{b}}$ and Fangyan Li, Xiangjiao Shen Chongqing University of Posts and Telecommunications, Chongqing 400065, China; aherder883@163.com, ${ }^{b} 378229119 @ q q . c o m$
}

Keywords: Wireless sensor network, DV-Hop algorithm, Localization

\begin{abstract}
Aiming at classical DV-Hop localization algorithm has a big positioning error in the situation that network nodes distribute unevenly, an improved DV-Hop algorithm is put forward. The anchor node's hop distance will be revised by hop counts in the parking lot of location model and hyperbolic positioning method is introduced to improve the positioning accuracy. The simulation results show that improved algorithm can greatly decrease the average localization error.
\end{abstract}

\section{Introduction}

With the development of wireless communications and sensor technology, wireless sensor networks (WSN) is widely used in daily life. In wireless sensor networks, location information is significant to monitoring data. Therefore, location technology is the key to process the collected data and achieve a variety of functions[1].

At present, many scholars have applied lots of methods, such as MPSO-DV-Hop[2], to position in the WSN [3]. According to the way of measure distance between nodes, positioning algorithm can usually be divided into rang-based algorithm and rang-free algorithm [4]. Zuo et al. [6] put forward an anchor node selection algorithm that can make the error lower. These algorithms' anchors and unknown nodes distribute randomly and are unsuitable on the situations that anchor nodes at the sides of unknown nodes. In this paper, we established the model of indoor parking to locate the place of car by wireless sensor networks.

\section{Analysis And Algorithm Description Of DV-HOP}

\section{The Original DV-HOP Algorithm}

DV-HOP algorithm expresses the distance between the unknown node and the anchor node by calculating the product of their hop count and the average hop distance of networks. The whole arithmetic processed could be concluded as follows.

Step 1: The hop counts between the unknown node and anchor node was obtained by the distance vector routing, and the information was transmitted cover the whole network by flooding.

Step 2: As an adjusted value, the average hop distance would be achieved after all information be given.

Step 3: The unknown node's coordinate will be obtained by using the three-side method and maximum likelihood.

The Error Analysis of DV-HOP Algorithm

The original DV-HOP algorithm's error was caused by the following situations:

1) The original DV-HOP's minimum hop count was achieved by the connectedness of network. 2) In original DV-HOP algorithm, the distance between the unknown node and the anchor node was achieved by calculating the product of their hop count and the average hop distance of networks.

\section{DV-HOP Algorithm Improvement}

The original DV-HOP algorithm sets the hop distance to equal for each unequal length jump, without taking the actual distance from a single jump into consideration. The following figure is the results of using original DV-HOP localization algorithm to locate the network model. 


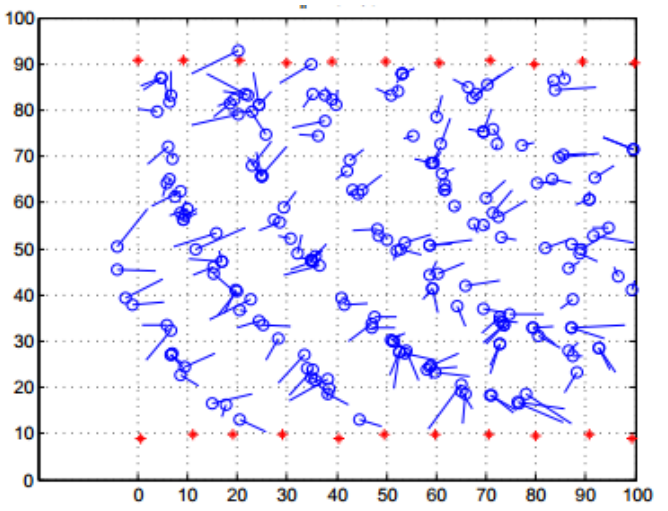

Fig. 1 The Results of Original DV-HOP Localization Algorithm

\section{Fixed The Calculation For Region Anchor Nodes}

The network model in this paper, the large anchor nodes hops will reduce the accuracy of the positioning deviation. Setting the hop threshold is hmin, when the hop threshold is larger than hmin. The hop distance outside the threshold is calculated as following.

$$
\text { HopSize }_{j}=\text { HopSize }_{j} \times \sum_{j}^{M} h_{j} / \sum_{1}^{m} h_{i j} \quad j \in\left(h_{i j}>h_{\text {min }}\right)
$$

In which, $h_{i j}$ is the hops from unknown node to the anchor node, $h_{\min }$ is the hop threshold, $M$ is the number of the anchor nodes whose hops is larger than threshold value. $m$ is the number of all anchor nodes. Anchors within the hop threshold can be based upon the original position algorithm to locate the position of anchor nodes, and the position coordinates is $\left(x_{e m}, y_{e m}\right)$. The formula of locating the unknown node's deviation is as follows.

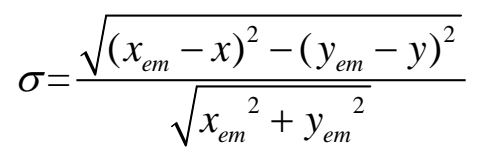

The hop distance by anchor nodes Improved is described as follows:

HopSize $_{i}=$ HopSize $_{i} \times(1+\sigma)$

\section{Hyperbolic Position Measurement}

Trilateration or the maximum likelihood method of Original DV-HOP algorithm has the part of equation group subtraction, then produce new deviation based on the distance from unknown nodes to anchor nodes. While trilateration will led to positioning node close to the hypotenuse of right triangle, so this paper proposed to using improved hyperbolic measurement method to calculate the position of unknown nodes. In this way, the deviation can be reduced.

The formula $G b=H$ can be concluded.

$b=\left(G^{T} G\right)^{-1} G^{T} H$ can be obtained by the least squares method, and also concluded the coordinates of unknown nodes, which is $\left\{\begin{array}{l}x=b(1) \\ y=b(2)\end{array}\right.$

\section{SIMULATION TEST AND ANALYSIS OF RESULTS}

In order to assess the effectiveness of the proposed algorithm, by using of Matlab2010, the original DV-HOP algorithm, reference5 and the improved algorithm proposed in this paper is simulated with the same wireless sensor networks model. The average location error is used as an evaluation criterion for different kinds of algorithm which are described above. In the process of simulation, all nodes including unknown nodes and anchor nodes are deployed in a $100 \times 100$ rectangular area. The simulation environment of the wireless sensor networks is used in this paper as follow: 


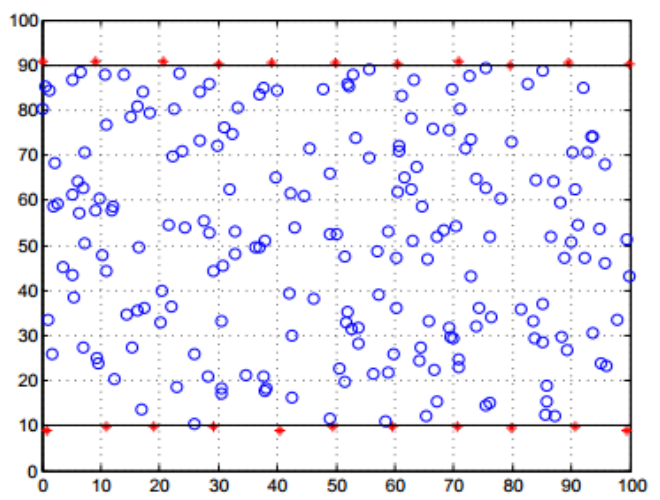

Fig.2 The Simulation Environment

\section{Change The Number Of Unknown Nodes}

On the basis of the wireless sensor network model we established above, we change the number of unknown nodes and keep anchors nodes constant. Then we choice the communication radius is $20 \mathrm{~m}$.

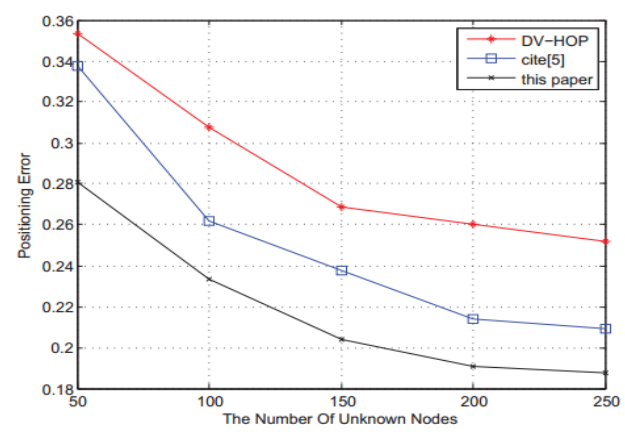

Fig.3 the number of unknown nodes

As can be seen from Fig3, with the increase of unknown nodes, the error rate of calculating unknown nodes coordinates is decreased. But we can see that the error rate is calculated by the improved algorithm proposed in this paper is lower than others that are referred to significantly and stabilizing. When the number of nodes increased, positioning error is relatively stable. In this paper, the positioning error rate is calculated by improved algorithm than the original DV-HOP algorithm decreased by about $10 \%$ and document 13 lower than about $6 \%$.

\section{Change Communication Radius}

On the basis of the wireless sensor network model we established above, we change the communication radius of nodes from $20 \mathrm{~m}$ to $40 \mathrm{~m}$ and keep unknown anchors nodes constant.

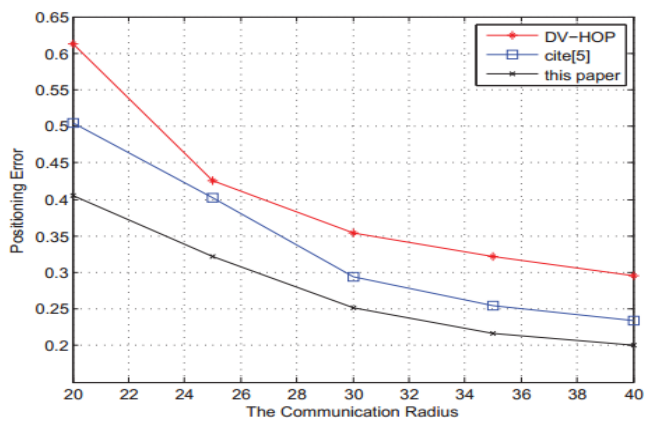

Fig.4 communication radius

As can be seen from Fig.4, with the increase of communication radius from $20 \mathrm{~m}$ to $40 \mathrm{~m}$, the error rate of calculating unknown nodes coordinates is decreased stably. But we can see that the error rate is calculated by the conventional DV-HOP algorithm from $61.2 \%$ down to $30 \%$ from $50 \%$ while the document 13 dropped to about 23\%. In this paper, the improved algorithm positioning error rate is dropped from $32.5 \%$ to $20 \%$, and compared to an overall reduction of about $15 \%$. 


\section{Summary}

This paper analysed the shortcomings of original DV-HOP algorithm and obtain the distance between anchor and unknown node by adjusting the hop counts. In addition, hyperbolic positioning method was introduced to improve the positioning accuracy. The simulation results show that the algorithm we proposed can reduce the positioning error effectively. Compared with classical DV_Hop and the algorithm put forward by Zuo et al. [5], the proposed algorithm's positioning error decreased about $12 \%$ and $6 \%$ respectively. In future work, we will focus on resolving the issues such as isolated nodes and calculation is complex and so on.

\section{References}

[1] SayedAH,Tarighat A,Khajehnouri N. Network-based wireless location: challenges faced in developing techniques for accurate wireless location information ,J.IEEE Signal Processing Magazine,2005,22( 4) : 24-40.

[2] ZHOU Tianqi. Node localization of wireless sensor network based on MPSO-DV-Hop, J. Computer Engineering and Applications.2013, 49 ( 23 ): 52-55.

[3] XIA Shaobo. Improvement on DV-Hop localization algorithm in wireless sensor networks. Journal of Computer Applications, J.2015, 35(2):340 344.

[4]Dragos Niculescu, Badri Nath. DV based positioning in ad-hoc networks, J .Telecommunication Systems, 2003, 22(1): 267-280.

[5] ZUO Wen-jun , YE Zhi-hui .An Improved DV-Hop Algorithm Based on Anchor Node Filtering, J. Computer and Modernization. 2014.225(5): 1 5.

[6]NiculescuD NathB. Adhoc positioning system( APS) using AoA[C]. Proceedings of IEEEINFOCOM 2003. SanFrancisco: IEEEComputer andCommunications Societies, 2003: 1734-1743.

[7] HUANG Dekai. Improved DV-Hop Algorithm Based on Hop Estimation, J. Computer and Modernization. 2012,208(12):4 7.

[8]WEN Jiangtao. Improved DV-Hop Location Algorithm Based on Hop Correction, J. Chinese Journal of Sensors and Actuators.2014,27(1):113 117. 